\title{
Dr. Georges Morel
}

(1921-2019)

El doctor Georges Morel nos dejó a los 98 años, silenciosa y suavemente, a la imagen de su vida profesional comprometida en cuerpo y alma con los niños. Las cualidades que mejor lo definían eran la calma, la serenidad, la dedicación y la entrega. Todos los que lo conocieron resaltaron siempre su

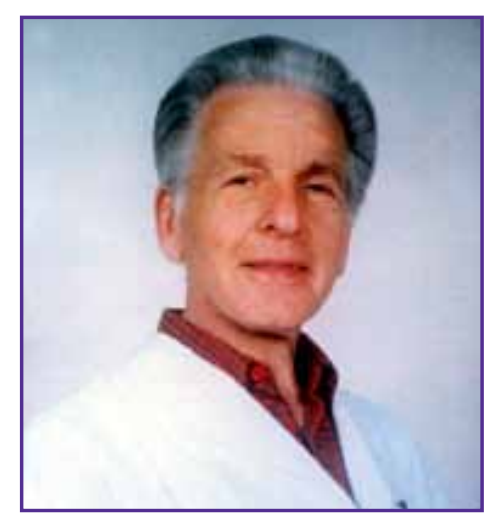
hombría de bien y su sabiduría. Al decir de uno de sus antiguos Residentes argentinos, "era un hombre admirable y de un gran humanismo: no solamente poseía la 'belleza del gesto quirúrgico', sino también el 'hermoso gesto humano"”.

Georges Morel nació el 17 de febrero de 1921, en Lille, Francia; hermano mayor de 6, su niñez y adolescencia estuvieron marcadas por las dificultades económicas de su padre (que había sido soldado en la "Gran Guerra" [1914-1918]) y por la afirmación precoz de su vocación: ya a los 7 años había decidido ser médico. A pesar de las dificultades, tuvo una niñez que se desarrolló en una familia feliz donde existía una gran unidad y armonía entre los hermanos, relación que se mantuvo hasta el final de sus días con las dos hermanas que aún le sobreviven.

Finalizado el Liceo, y aprobado el Certificado de estudios Físicos, Químicos y Biológicos (prerrequisito para entrar en la Facultad de Medicina), continuando con su objetivo, comenzó sus estudios de Medicina en la Universidad Católica de Lille. Si bien le tocaron los años negros de la II Guerra Mundial, tuvo la suerte de trabajar como disector de Anatomía y de poder vivir como interno en diferentes hospitales, cercano a los enfermos -"al pie del enfermo" como le gustaba decir-. En sus años de ayudante de cátedra y disector, adquirió un gran conocimiento de la anatomía que sabría aprovechar años más tarde para mejor abordar una cadera luxada o un pie bot. No pudo concretar su formación en Cirugía a causa de la guerra, pero fue durante esas estadías como interno que residió, por primera vez, en el Institut Calot de Berck, teniendo una de sus mejores experiencias y creando vínculos afectivos con las personas de allí. Conoció también en esta época a su novia -Jaqueline Deram- y se casó con ella en agosto de 1948.

Terminada la contienda, logró un puesto de Interno (Residente) en Cirugía, en la localidad de Oignies (en la Región Nord-Pas de Calais) donde trabajó a destajo por la salud de los mineros de la región.

Partió luego a Camerún (África) para trabajar en el Hospital de Efok como cirujano para una organización de caridad católica (Ad Lucem), pero debido a un paludismo muy complicado de su esposa, regresó a Francia al cabo de 7 meses con ella y su pequeña hijita. Se instaló entonces en octubre de 1950, en Berck aceptando una propuesta de trabajo del Institut Calot. Berck-sur-mer, un pequeño pueblo sobre el Paso de Calais (Mar del Norte), era desde la segunda mitad del siglo XIX, sitio de florecimiento de diferentes Hospitales dedicados especialmente a las enfermedades osteoarticulares, entre ellos, el Institut Calot (inicialmente el Institut d' Orthopédie) fue uno de los más emblemáticos. Allí se encontró entonces con Yves Cotrel, agregándose a esta dupla Jean Duriez -quien se encargaría de la Reumatología y la Investigación Básica- y Gastón Héripret -el Radiólogo que tanto ayudó y creó en "el Calot" -. Juntos formaron "la nueva banda de los 4 de Berck", como afectuosamente se los conoce en el ámbito científico-médico francés -por comparación con Victor Ménard, François Calot, Jacques Calvé y Etienne Sorrel, adalides del progreso científico-médico en Berck a principios del siglo XX-. Este nuevo equipo de 4 "grandes" - del que tanto participó Georges Morel- fue sagazmente dirigido por el Profesor Jean Cauchoix, y su trabajo científico produjo innumerables e importantes investigaciones a partir de 1955 (Figura 1). Morel y Cauchoix establecieron en el Calot una fuerte amistad que duró toda la vida de ambos...

Dr. PATRICIO MANZONE • manzonepatricio@ hotmail.com (ID

Cómo citar este artículo: Manzone P, Santodomingo J. Obituario. Dr. Georges Morel. Rev Arg Asoc Ortop Traumatol 2019;84(4):437-441. http://dx.doi.org/10.15417/issn.18527434.2019.84.4.1017 


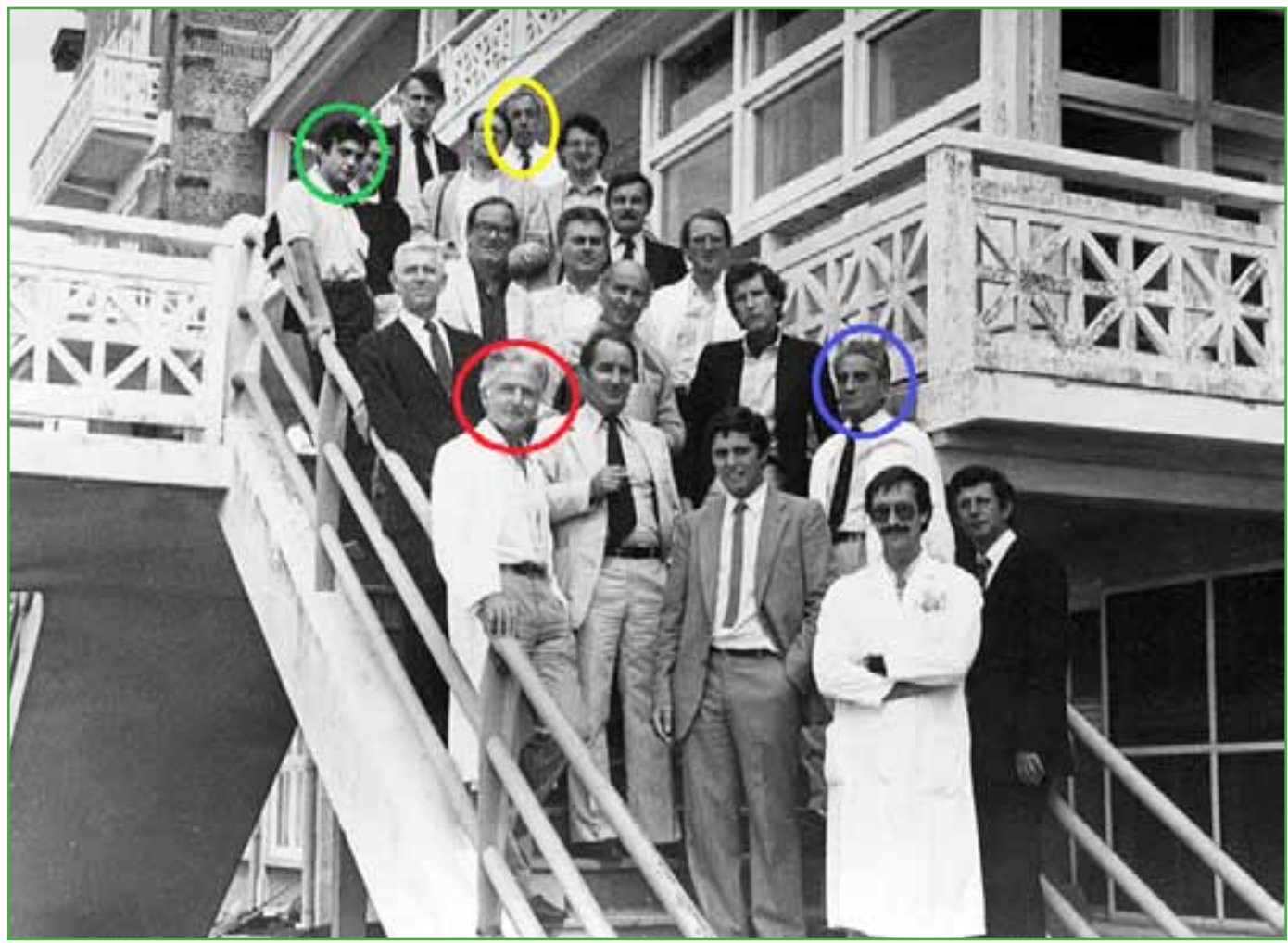

Figura 1. Grupo de médicos del Institut Calot: el doctor Morel (círculo rojo), el doctor Heripret (círculo amarillo), el profesor Cauchoix (círculo azul) y el doctor Christian Morin -sucesor del doctor Morel(círculo verde).

Calmo y sereno en su actuar, Morel era, sin embargo, inquieto, curioso, autocrítico, responsable y profundo en su faz científica intelectual. Sabedor de las limitaciones en su formación inicial en Ortopedia Infantil, realizó numerosos cursos y estadías en el Black Notley Hospital (en el condado de Essex, Inglaterra) -en el Servicio del doctor Wilkinson- y en el Royal National Orthopaedic Hospital de Londres, Inglaterra. También fue asiduo visitante del Servicio de Ortopedia del profesor Merle d'Aubigné en el Hôpital Cochin, de París.

Morel, en su estancia laboral en el Institut Calot hasta febrero de 1986 -cuando se jubiló-, se dedicó "con total modestia pero con una devoción sin parangón al bienestar del niño tullido o malformado", según palabras del propio profesor Cauchoix; lo que lo llevó a ser un gran nombre en la Ortopedia Pediátrica Francesa. Tal era su devoción que el día de su cumpleaños 65 (17 de febrero de 1986), justo antes de jubilarse, realizó su última osteotomía de pelvis que se había comprometido a efectuar.

Amaba apasionadamente por igual su oficio y a los niños que cuidaba: sea en el consultorio como con los internados, el pequeño paciente y sus padres estaban en el centro de sus preocupaciones, haciéndoles sentir que, en la habitación, eran las personas más importantes; y esto, cualquiera fuera su condición social, raza o credo.

Era un clínico notable y un cirujano sereno en cuyas manos "todo parecía fácil y sin peligro". Verlo operar era un placer: hemostasia perfecta, respeto de los planos de disección, ausencia de cualquier gesto brusco, prudencia en las maniobras de corrección y la técnica "no touch" (nunca una gota de sangre tocaba sus guantes).

Georges Morel no era Profesor Universitario; sin embargo, fue un MAESTRO y un modelo a seguir para sus discípulos y alumnos que descubrieron a su lado la Ortopedia Pediátrica. Disfrutaba especialmente enseñar a sus Residentes reunidos junto a un negatoscopio a la noche, después de una muy larga jornada quirúrgica. El Institut Calot recibía anualmente becarios de todos los rincones del mundo, pero cuatro orígenes predominaban ampliamente: el Servicio del profesor Cauchoix en el Hospital Beaujon (París), los becarios del Líbano, los médicos de México y la FILIAL ARGENTINA. En efecto, una legión de cirujanos ortopedistas argentinos gozaron del beneficio de estas enseñanzas (Figura 2). Fue guía de varias generaciones... 


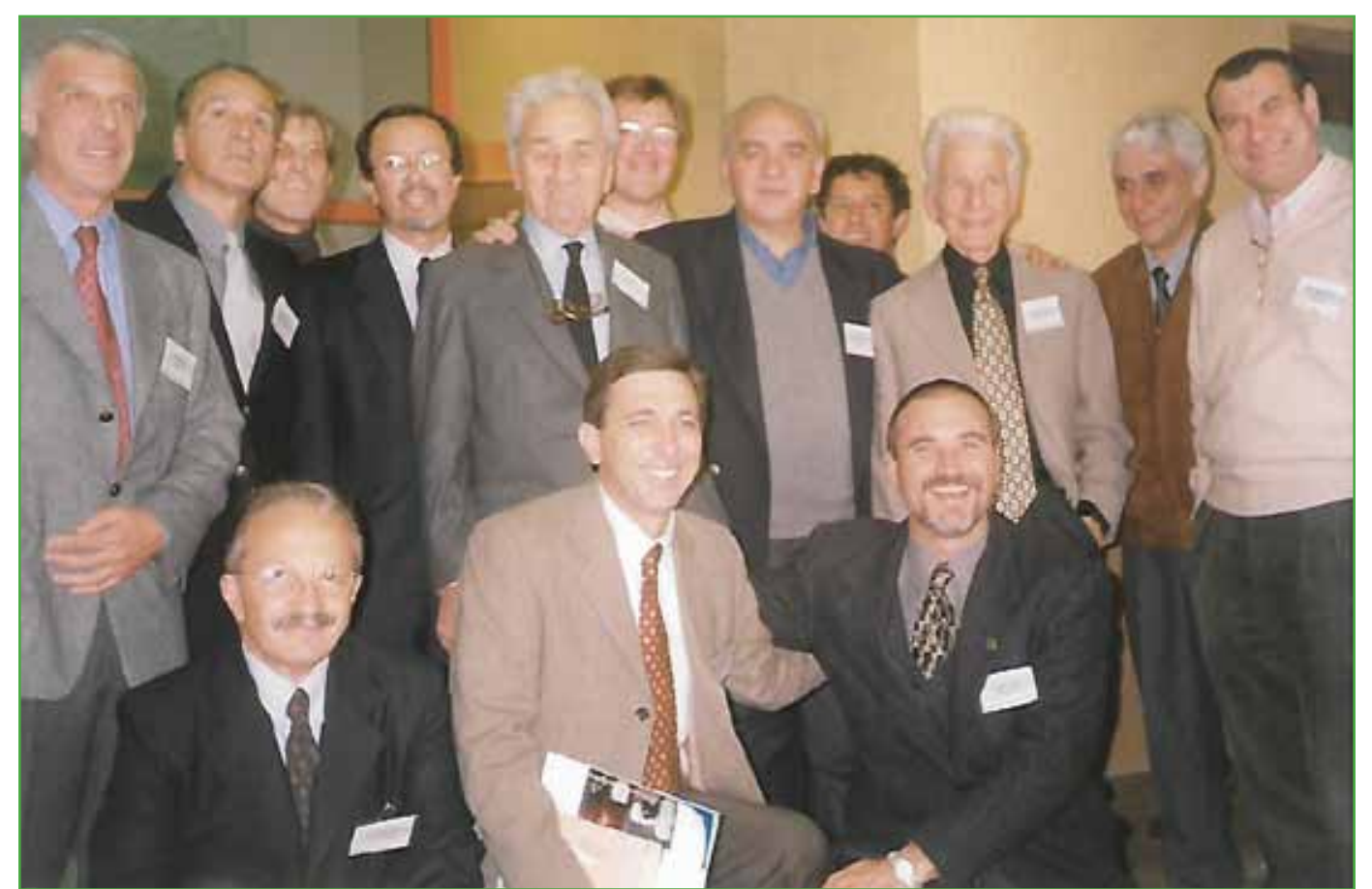

Figura 2. Un grupo parte de la "Legión Argentina" de cirujanos ortopedistas formados en posgrado, en el Calot, junto al profesor Jean Cauchoix y al doctor Georges Morel. Foto tomada en el año 2000, durante el festejo de los 100 años del Institut Calot.

Una o dos noches por semana los residentes eran invitados a la oficina del "patrón" para descubrir historias clínicas impecablemente llevadas y con un seguimiento digno de ser mostrado: una radiografía de pelvis de un adulto absolutamente normal, pero con una antigua radiografía de un niño de 2 años con una luxación de cadera alta, o una radiografía de raquis normal de un joven de 20 años que, a los 3 años, mostraba una "escoliosis de comienzo temprano" (según su nomenclatura actual) que ya tenía $50^{\circ}$ y que parecía destinada a una artrodesis segura.

Sus temas predilectos fueron muchos: la Luxación de Cadera después de la edad de la marcha (con resultados a largo plazo sin igual), el Pie Bot varo equino del niño mayor, el tratamiento ortopédico de las Escoliosis Infantiles (con trabajos en colaboración con Cotrel sobre los yesos correctores y con Min Metha sobre la posibilidad de curación de las "progresivas benignas"), el tratamiento de las dismetrías de miembros inferiores, el abordaje de la Osteogénesis Imperfecta (la ortesis neumática de su invención sirvió durante muchos años para el cuidado de estos niños tan frágiles). ${ }^{1-5}$

Revisaba con una constancia pasmosa las historias clínicas de sus antiguos pacientes, sobre todo para intentar comprender lo que salió mal y encontrar la solución. Fue esta revisión meticulosa de las historias clínicas la que lo llevó a un profundo convencimiento de que el tratamiento clásico -indiscutible por entonces (eran los años 60) - de la Luxación del Desarrollo de la Cadera descubierta Tardíamente (después de la marcha) por reducción ortopédica seguida de osteotomía femoral de desrotación-varización no daba al final del crecimiento resultados exitosos. El descubrimiento fortuito -en un Congreso- de una operación nueva descrita entonces por un cirujano canadiense -Robert Salter, quien era orador en dicho Congreso- fue para Morel una revelación: era a esta osteotomía de pelvis -y no a la sacrosanta osteotomía de fémur-a la que se debía confiar la estabilización y el tratamiento de la displasia después de la reducción ortopédica de la luxación. Hacer aceptar a la comunidad ortopédica francesa esta nueva manera de ver las cosas no fue fácil: necesitó de mucho coraje (incluso "temeridad" según uno de los espectadores de la época) al presentar, en noviembre de 1970, su experiencia con sus 100 primeros casos en el Congreso anual de la SOFCOT (Sociedad Francesa de Cirugía Ortopédica y Traumatológica) ${ }^{6}{ }^{6}$ El moderador de su exposición era Pierre Petit, maestro indiscutido en esos tiempos de la Ortopedia Pediátrica francesa! Y necesitó muchos años más para que sus ideas fueran aceptadas y apreciadas en toda su magnitud..$^{7-9}$ Es conocida la historia de que un día, en un Congreso en México, como orador invitado junto a Salter, el mismo canadiense dijo que los resultados que Morel obtenía eran mejores que los suyos y que lo había superado en el dominio de su técnica... 
Otra contribución mayor de Georges Morel fue en el tratamiento ortopédico de las Escoliosis Idiopáticas del niño pequeño ("early-onset scoliosis"): en 1974, durante el "meeting" anual del GES (Grupo de Estudios de la Escoliosis) presentó 10 casos de escoliosis infantiles evolutivas en las que el tratamiento ortopédico había dado prueba de una eficacia excepcional. Esta comunicación desafió y conmocionó tanto al "mundo de la escoliosis" que la experiencia de "Morel from Berck-Plage"será hasta citada en la prestigiosa obra de John Moe y Robert Winter (Scoliosis and Other Spinal Deformities). ${ }^{10}$ Tampoco pasó inadvertida en Gran Bretaña, ${ }^{11,12}$ a tal punto que la doctora Min Mehta -escolióloga emérita- lo visita en el Institut Calot para estudiar sus casos y su técnica, refinar así sus conceptos y método de tratamiento ortopédico. ${ }^{13}$ Método que hoy día es aún uno de los pilares del tratamiento de las escoliosis de comienzo temprano..$^{14,15}$

Pero si bien las responsabilidades profesionales y científicas lo absorbían de manera intensa, Georges Morel fue un padre ejemplar, siempre presente y muy cercano a sus hijos (Martine, Bruno, Laurence y Charlotte), y luego a sus nietos. ¡Y no solamente los domingos que eran consagrados a la familia! También durante la semana, en pequeñas acampadas durante algún fin de semana, en vacaciones de familia, incluso en vacaciones de papá e hijos solos (¡sin la mamá! cuando ella necesitaba de un "descanso de familia"), al menos, una vez al año cuando las aventuras harían las delicias de todos con recuerdos imborrables...

Llegada su jubilación dio vuelta la página sin nostalgia, habiendo tenido la suerte de realizarse en un oficio apasionante desde un doble punto de vista profesional y humano; y habiendo escogido la vida que quiso llevar, también tuvo la suerte de poder elegir a su sucesor, el doctor Christian Morin (Figura 3). Lejos de "quedarse quieto", se movió más, pero en otro camino: el de compartir con artistas (pintores, dibujantes) que visitó sistemáticamente durante años en el barrio latino de París y el de compartir con su familia que se agrandaba (nietos y bisnietos).

En fin, Georges Morel nos dejó este año, el 20 de junio pasado, luego de una vida plena y fructífera. Sin embargo, seguirá vivo en los corazones y mentes de sus hijos, nietos, bisnietos, y de sus innumerables discípulos a lo largo y ancho del mundo que supieron apreciar sus enseñanzas para mejorar la calidad de vida de los niños. QEPD

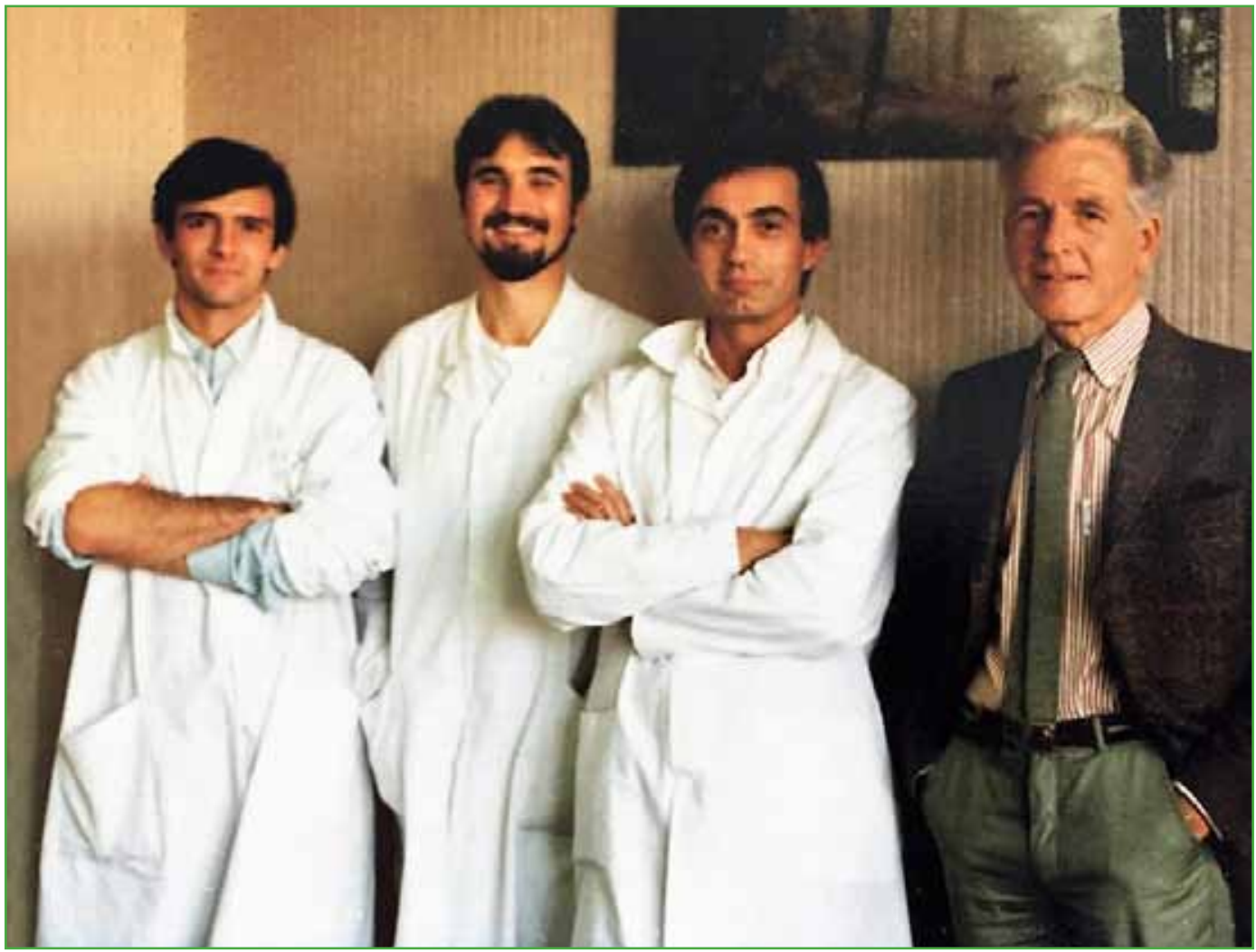

Figura 3. El doctor Georges Morel, junto a su sucesor el doctor Christian Morin, al doctor Henry-François Parent (expresidente de la Sociedad Francesa de Cirugía del Raquis [SFCR]) y uno de los autores. Foto de 1989. 


\section{BIBLIOGRAFÍA}

1. Morel G. The treatment of congenital dislocation and subluxation of the hip in the older child. Acta Orthop Scand 1975;46:364-99. PMID: 1146522

2. Morel G. La correction chirurgicale du pied bot du grand enfant. En: Carlioz H, Pous JG. Cahiers d'enseignement de la SOFCOT No 3 Le pied bot varus équin. Paris: Expansion Scientifique Française; 1976:91-103.

3. Cotrel Y, Morel G. La technique de l'E.D.F dans la correction des scolioses. Rev Chir Orthop 1964;50:59-75.

4. Morel G, Morin C. Technique simplifiée de l'allongement extemporané du fémur chez l'enfant. Chir Pediatr 1986;27(2):91-3. PMID: 3742699

5. Morel G. Un nouveau type d'appareillage orthopédique: L'appareillage à attelles pneumatiques. Rev Chir Orthop Reparatrice Appar Mot 1971;57(5):409-14. PMID: 4257960

6. Morel G. L'ostéotomie de Salter dans le traitement de la malformation luxante de la hanche après l'âge de la marche. Rev Chir Orthop Reparatrice Appar Mot 1971;57(Sup 1):175-83. PMID: 4252753

7. Morel G. Le traitement de la hanche luxée de l'âge de la marche à 5 ans. Acta Orthop Belg 1990;56(1 Pt B):237-49. PMID: 2382557

8. Rampal V, Sabourin M, Erdeneshoo E, Koureas G, Seringe R, Wicart P. Closed reduction with traction for developmental dysplasia of the hip in children aged between one and five years. J Bone Joint Surg $\mathrm{Br}$ 2008;90(7):858-63. https://doi.org/10.1302/0301-620X.90B7.20041

9. Morin C, Bisogno J, Kulkarni S, Morel G. Treatment of late-presenting developmental dislocation of the hip by progressive orthopedic reduction and innominate osteotomy. Our results with more than 30 years of follow up. $J$ Child Orthop 2011;5(4):251-60. https://doi.org/10.1007/s11832-011-0346-2

10. Moe J, Winter R, Bradford D, Lonstein J. Scoliosis and other spinal deformities. Philadelphia: WB Saunders Company; 1976:93.

11. Dickson RA, Leatherman KD. Cotrel traction exercises, casting in the treatment of idiopathic scoliosis. Acta Orthop Scand 1978;49(1):46-8. https://doi.org/10.3109/17453677809005722

12. Dickson RA. Conservative treatment for idiopathic scoliosis. J Bone Joint Surg Br 1985;67(2):176-81. PMID: 3872301

13. Mehta MH. Growth as a corrective force in the early treatment of progressive infantile scoliosis. J Bone Joint Surg Br 2005;87(9):1237-47. https://doi.org/10.1302/0301-620X.87B9.16124

14. D’Astouz JL, Sanders JO. Casting and traction treatment methods for scoliosis. Orthop Clin North Am 2007;38(4):477-84. https://doi.org/10.1016/j.ocl.2007.03.006

15. Morin C, Kulkarni S. ED plaster-of-Paris jacket for infantile scoliosis. Eur Spine J 2014;23(Suppl 4):S412-18. https://doi.org/10.1007/s00586-014-3336-5 